\title{
INDOCYANINE GREEN ENHANCED LAPAROSCOPIC CHOLECYSTECTOMY IN ACUTE PATIENTS: DO WE NEED A REVISION OF THE SAFETY PARADIGMS?
}

\author{
Catalin Alius $^{1}$, Eugen-Sebastian Gradinaru' ${ }^{1}$, Adriana Elena Nica ${ }^{2}$ \\ ${ }^{1} 4^{\text {th }}$ Department of Surgery, Bucharest University Emergency Hospital \\ ${ }^{2} 2^{\text {nd }}$ Intensive Care Unit, Bucharest University Emergency Hospital
}

\begin{abstract}
Introduction. Rapid developments in medical technology have allowed the incorporation of Indocyanine green (ICG) fluorescent cholangyography in the surgical technique armamentarium. The visualization of the biliary anatomy with augmented reality devices during surgery reduces complications and offer the perspective of challenging the safety paradigms which prohibited surgery in certain acute biliary conditions.

Materials and methods. 43 consecutive patients were enrolled in a prospective interventional study and randomly divided into a cohort of 19 patients who had ICG injected prior to laparoscopic cholecystectomy and a cohort of 23 patients who received no fluorescent dye prior to surgery. In the ICG lot a Near Infrared Fluorescent System was used for the acquisition of fluorescent data in order to produce real time augmented reality imaging (ICG fluorescent cholangiography). The surgical technique and the indications for surgery were the same for the same in both cohorts of patients.

Results and discussion. The cohort of patients receiving ICG had no complications and the mean operating time was 10 minutes less. The biliary anatomy was identified immediately in the ICG cohort with a specificity of $89.4 \%$ for the common bule duct and $73.6 \%$ for the cystic duct. In the non ICG cohort $21 \%$ of the CBDs and $43.4 \%$ of the cystic ducts were identified with difficulty during the procedure.

Conclusion. We demonstrated in a small cohort of patients that early laparoscopic cholecystectomy is safe and can be performed quicker with the aid of fluorescent dyes. In order to challenge the safety paradigms around the early laparoscopic cholecystectomy a larger study is necessary.
\end{abstract}

Keywords: Indocyanine Green (ICG), laparoscopic cholecystectomy, fluorescence

\section{INTRODUCTION}

Technologies in the medical field have known an unprecedented development over the past two decades with various devices and methods being translated into clinical practice from different engineering areas. Among these is the recent introduction of commercially available Near Infrared (NIR) Fluorescence Imaging Systems for visualization of obscure anatomical structures and assessment of tissue function and viability. By intravenous administration of small amounts of fluorophores and by overlapping standard images with NIR acquired data one can obtain real time information about the very substance of the tissues such as: precise location of tubular structures which would normally be inaccessible to the naked eye CBD (common bile duct), delineation of surgical resection margins, assessment of invasion, accurate location of metastatic deposits and quality of tissue perfusion $(5,6)$.

The introduction of laparoscopic cholecystectomy by the German surgeon Erich Mühe of Böblingen generated an increase in the bile duct injuries compared to the open approach (1). Cuschieri predicted an increased risk of bile duct injuries immediately following the introduction of the laparoscopic technique but the rate of complications remained steady over two decades despite the growing number of properly trained surgeons (2). The incidence of common bile duct (CBD) injuries in laparoscopic cholecystectomy is between $0.3 \%$ and $0.7 \%$ with no less than $0.4 \%$, but still three times higher than in open cholecystectomy (2). In the state of New York after decades of operating 
laparoscopic cholecystectomies, Halbert publishes in 2017 the first paper to report that biliary complications could be reduced to values similar to those reported with the use of the open approach if sufficient and structured training is provided (4). Further more because of the high incidence of anatomical variations of the cystic artery and bile ducts associated with oedema of the tissues in acute inflammatory conditions, early laparoscopic cholecystectomy for acute cholecystitis is associated with higher risk of morbidity and thus not recommended in many regions of the Globe (7).

We believe that the paradigm of prohibiting early surgery for acute cholecystitis could be reevaluated in the light of this recent technology which could reduce the complication risks to values similar to or even lower to those encountered with the open cholecystectomy technique and could also compensate for the lack of extensive laparoscopic training.

\section{MATERIALS AND METHODS}

\section{Patients}

We conducted a prospective interventional study on 42 consecutive patients who presented with acute cholecystitis at Bucharest University Hospital. The study was approved by the ethical committee and was conducted with strict adherence to the principles of the Declaration of Helsinki. Eligibility criteria consisted of: age above 18 years and a diagnosis of acute cholecystitis, biliary pancreatitis or complicated cholelithiasis. Exclusion criteria were represented by: pregnancy, allergy to iodine, thyroid disease or previous allergic reaction to contrast. We collected demographical data from all the 42 patients enrolled and randomization for assignment to the ICG and non-ICG lots was performed.

\section{Indocyanine Green (ICG)}

ICG is a fluorescent dye developed almost half a century ago and used in surgery because of its properties of emitting at $830 \mathrm{~nm}$ in the NIR spectrum of light. When injected intravenously this sterile, anionic, tricarbocyanine molecule mixes with the blood and binds to proteins. It has a very short half life into the bloodstream (3-5 minutes) and it is excreted into the bile almost entirely after 10-15 minutes having no known metabolites. Because it concentrates into the bile, has a very short half life in the blood stream and a low toxicity, ICG has become the fluorescent contrast of choice for florescent intraoperative cholagiography $(9,10)$.

\section{NIR Imaging System}

We used a PINPOINT endoscopic fluorescence imaging system By NOVADAQ (Canada) PC 900 with a high definition camera, and a 45 degrees telescope. The system is a laparoscopic device with an additional excitation light emitted at $805 \mathrm{~nm}$ at $20 \mathrm{~Hz}$ and optical filters for visualization of the NIR fluorescence. The images obtained in the NIR spectrum are converted into images in the visible spectrum and can be added over conventional images in real time. After allocation to specific lots the ICG patients received $0.02 \mathrm{mg}$ of ICG per $\mathrm{kg}$ if their BMI was under $25,0.04 \mathrm{mg} / \mathrm{kg}$ if the BMI was between 25 and 30 and $0.06 \mathrm{mg} / \mathrm{kg}$ if the BMI was above 30 . We started to inject at 45 minutes prior to camera port insertion and first camera look with ICG was recorded after 45 minutes, followed by a second look at 90 minutes, and/or at the end of the operation (if duration less than $90 \mathrm{~min}$.) after the extraction of the gallbladder was completed. In order to assess the quality of the fluorescence we used a subjective scale designed by Zarrinpar et al. in a clinical study that was imagined in order to establish the necessary amount of ICG needed for optimum visualization of the structures (8).

We marked the visualization of CBD and of the cystic duct separately with marks from 1 to 5 as follows: $1=$ no improvement, $2=$ marginally improved, $3=$ sufficiently improved, $4=$ well improved, $5=$ greatly improved. The assessment of the intensity of the fluorescence was performed by the first operator and by the assistants who were blinded to the dosage but not to the timing of injection. The nonICG cohort had laparoscopic cholecystectomy with conventional imaging systems and received no fluorescent dye. At the end of the study we analyzed the duration of the surgery in the two cohorts, the comorbidities associated with the surgery in the two lots, demographic data and the ease of $\mathrm{CBD}$ and cystic duct recognition.

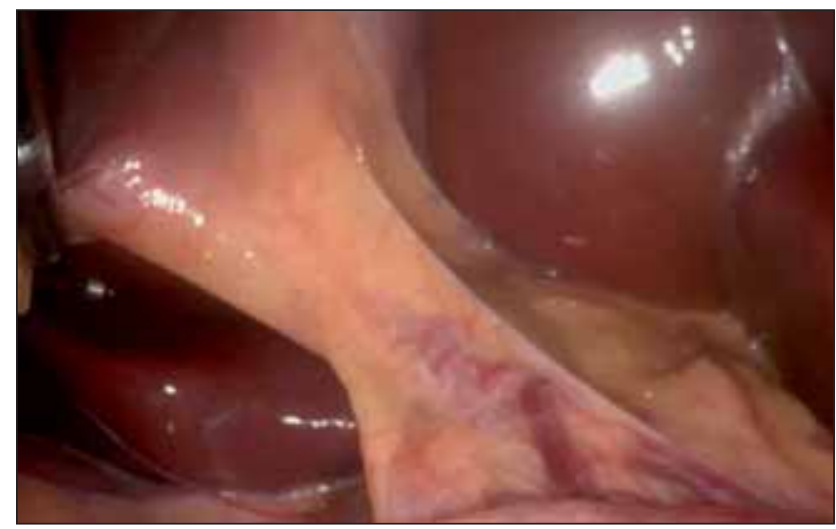

FIGURE 1. Conventional imaging of the cystic duct and the $C B D$ under fatty tissue 


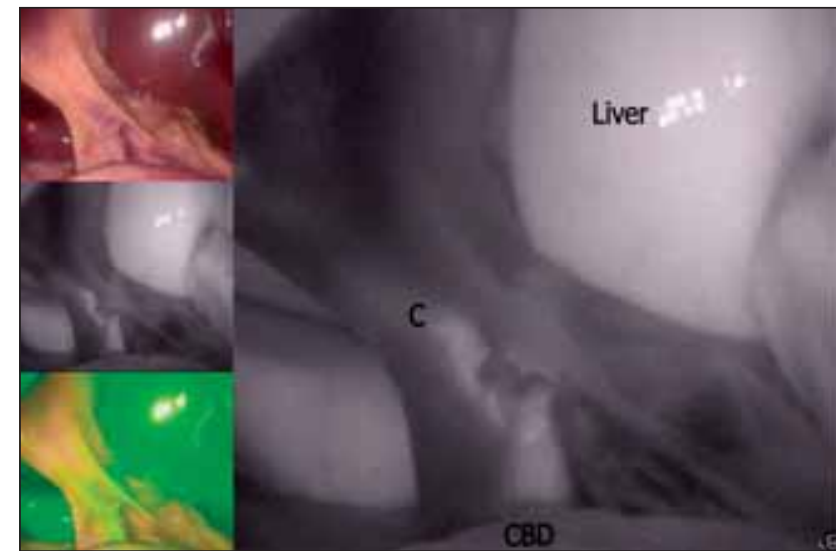

FIGURE 2. Main image showing cystic and the CBD ducts in digitalized NIR imaging; Image in the upper left corner shows conventional imaging; Image in the lower left corner shows the biliary tree in augmented reality

\section{RESULTS AND DISCUSSIONS}

\section{Results}

The patients characteristics are detailed in Table 1 and a comparison of the main data acquired during surgery in Table 2 . In the ICG group 11 patients $(57.89 \%)$ were females and 8 patients $(42.1 \%)$ were males. The median age was 58+/_ 16 years and the mean BMI was 29+/2.9. The CBD was visualized immediately in 17 patients (specificity $89.4 \%$ ) and the cystic duct in 14 patients (specificity $73.6 \%$ ). The mean operating time was $65+/ 14 \mathrm{~min}$. In the non ICG group 13 patients were females $(52.17 \%)$ and 11 patients were males $(47,82 \%)$. The median age in this lot was 58+/ 15 years and the mean BMI was $28.5+/ 2.3$. The mean operating time was $75+/-$ 15 minutes. We used a Students T test to determine statistical significance between groups and the result showed a "p" value less than 0.05. Data was analyzed using Excel and Minitab software.

\section{DISCUSSIONS}

The small cohort of patients receiving early laparoscopic cholecystectomy did not suffer complications associated with the surgery.
Although rare, injuries to $\mathrm{CBD}$ during laparoscopic surgery can be reduced by better visualization of the biliary tree. This is demonstrated by immediate recognition of the $\mathrm{CBD}$ in the ICG lot compared with the non-ICG patients in whom careful dissection and permanent reevaluation was needed to avoid damage. This is reflected in operating times lower than those reported in the non ICG group (median 65 minutes) and identification of the CBD in 17 out of 19 patients within seconds from insertion of the camera telescope (specificity $89.4 \%$ ) and identification of the cystic duct in 14 patients with minimal traction and dissection (specificity 73.6\%). Furthermore we identified 2 anatomical variants in the ICG group and 1 in the non ICG. The variants are too small in number to have statistical power hence it is not reasonable to assume that the shorter operating time associated with the two ICG case is related to the use of the dye, further studies being needed to clarify this aspect. Applying the same rationale the only case of conversion was attributed to a local inflammatory mass with very high risk of biliary tree damage and there is no evidence to support that the use of ICG would have changed the decision to convert. The bigger doses utilized in patients with a high BMI might have added to the penetration of the NIR light and eased the identification of the anatomical structures, but concomitant studies suggested higher doses for better intraoperative view (8). We found the fluorescent intensity of the liver at 45 minutes a limiting factor of visualization at higher doses in a small trial we conducted prior to this larger study. Small doses were sufficient to delineate the anatomical structures.

\section{CONCLUSIONS}

The development of NIR assisted optical systems for laparoscopic surgery has been envisaged to solve the clinical problem posed by the limitation of the surgeons visual abilities. This new technology allows tissue penetration up to $1 \mathrm{~cm}$ with the use of NIR light and provides real time imaging

TABLE 1. Demographics of the participants

\begin{tabular}{|l|c|c|c|c|c|}
\hline \multicolumn{3}{|c|}{ ICG COHORT } & \multicolumn{3}{c|}{ NON ICG Cohort } \\
\hline BMI & Median & $29+/ 2.9$ & BMI & Median & $28.5+/ 22.3$ \\
\hline Gender & Male & $8(42.1 \%)$ & Gender & Male 11 (47,82\%) & $8(42.1 \%)$ \\
& Female & $11(57.89 \%)$ & & Female 12 (52,17\%) & $11(57.89 \%)$ \\
\hline Age & Median & 58 & Age & Median & 58 \\
& Range & $23-86$ & & Range & $34-82$ \\
\hline Indication & Acute cholecistitis & 13 & Indication & Acute cholecistitis & 15 \\
& Biliary pancreatitis & 5 & & Biliary pancreatitis & 6 \\
& Cholelithiasis & 1 & & Cholelithiasis & 2 \\
\hline
\end{tabular}


TABLE 2. Intraoperative variables

\begin{tabular}{|c|c|c|c|c|c|}
\hline \multicolumn{3}{|c|}{ ICG COHORT } & \multicolumn{3}{|c|}{ NON ICG COHORT } \\
\hline Duration & Median & $65 \mathrm{~min}+/$ 14 min & Duration & Median & 75min+/_15 min \\
\hline $\begin{array}{l}\text { Malformations } \\
\text { and time of surgery }\end{array}$ & $\begin{array}{c}\text { Aberant CBD } \\
\text { Aberant cystic artery }\end{array}$ & $\begin{array}{l}1 \\
1\end{array}$ & $\begin{array}{l}\text { Malformations and } \\
\text { time of surgery }\end{array}$ & Aberant cystic duct & 1 \\
\hline Complications & 0 & & Complications & & 1-conversion \\
\hline $\begin{array}{l}\text { Rapid CBD } \\
\text { identification }\end{array}$ & $\begin{array}{l}\text { Immediate in } 17 \\
\text { patients }\end{array}$ & $89.47 \%$ Spec & CBD identification & Difficult in 5 patients & $21 \%$ difficult \\
\hline $\begin{array}{l}\text { Rapid cystic duct } \\
\text { identification }\end{array}$ & Rapidly in 14 patients & 73.68\% Spec & $\begin{array}{l}\text { Cystic duct } \\
\text { identification }\end{array}$ & Difficult in 10 patients & $43.4 \%$ difficult \\
\hline
\end{tabular}

which demonstrates the local anatomy of the biliary tree and vessels (12-17). Although CBD injuries occur with a relatively low frequency they are severe complications with a mortality up to $11 \%$ and $70-80 \%$ are secondary to missidentification of the anatomy (11). We demonstrated in a small co- hort of patients that early laparoscopic cholecystectomy is safe and can be performed quicker with the aid of fluorescent dyes. A larger study could be able to challenge the prohibition promoted by various surgical centres in the world on early laparoscopic cholecystectomy for acute biliary conditions.

\section{REFERENCES}

1. Reynolds W.Jr. The first laparoscopic cholecystectomy. JSLS 2011; 5(1):89-94

2. Gigot J.F. Bile duct injury during laparoscopic cholecystectomy: risk factors, mechanisms, type, severity and immediate detection. Acta Chir Bel. 2003; 103:154-160

3. Vettoretto N., Saronni C., Harbi A. et al. Critical view of Safety During Laparoscopic Cholecystectomy. JSJS 2011; 15:322-325

4. Halbert, Pagkratis S., Yang J. et al. Beyound the learning curve: incidence of bile duct injuries following laparoscopic cholecystectomy normalize to open in the modern era. Surg. Endosc. 2016; 30(6):2239-43.

5. Gioux S., Soo Choi H., Frangiony J.V. Image Guided Surgery using Invisible Near infrared Light: Fundamentals of Clinical Translation. Mol. Imaging. 2010; 9(5):237-255

6. Boni L., David G., Mangano A. et al. Clinical applications of indocyanine green (ICG) enhanced fluorescence in laparoscopic surgery. Surg. Endosc. 2015; 29(7):2046-2055.

7. Nagral S., Anatomy relevant to cholecystectomy. J Minim Acces Surg. 2005; 1(2):53-58

8. Zarrinpar A., Dutson E., Mobley C. et al. Intraoperative Laparoscopic Near Infrared Fluorescence Cholangiography to Facilitate Anatomical Identification: When to Give Indocyanine Green and How Much. Surgical Innovation 2016;23(4);360-365

9. Hellan M., Spinoglio G., Pigazzi A. et al. The influence of fluorescence imaging on the location of bowel transaction during robotic left sided colorectal surgery. Surg Endosc 2014; 28(5):1695-702

10. Miwa M. The principle of ICG fluorescence mode. Open surgical Oncol J 2010; 2:26-28

11. Jablonska B., Lampe P. latrogenic bile duct injuries: Etiology diagnosis and management. World J Gastroenterol 2009; 15(33):4097-4104

12. Schols R.M., Bouvy N.D., Masclee A.A., Van Dam R.M. et al. Fluorescence cholangiography during laparoscopic cholecystectomy: a feasibility study on biliary tract delineation. Surg Endosc. 2013; 27:1530-1536

13. Buchs N.C., Hagen H.E., Pugin F. et al. Real time near infrared fluorescent cholangiography using indocyanine green during robotic singe site cholecystectomy. Int J Med Robot. 2012; 8:436-440

14. Askalaki D., Fernandes E., Wang X. et al. Indocyanine green (ICG) fluorescent cholangiography during robotic cholecystectomy: results of 184 consecutive cases in a single institution. Surg Innov. 2014;21:615-62. 